\title{
Hérnia de Richter em portal de laparoscopia: relato de caso
}

\author{
Richter's hernia in laparoscopy portal: case report \\ La hernia de Richter en el portal de laparoscopia: reporte de caso \\ Laura Dias Pereira ${ }^{1}$, Antonio Goulart Monteiro ${ }^{1}$, Nataly Gomes Tolentino ${ }^{2 *}$, Nathalia Ferreira Nunes².

\section{RESUMO} \\ Objetivo: Apresentação de quadro clínico de uma paciente que apresentou Hérnia de Richter, uma condição \\ rara. Detalhamento do caso: Paciente, 64 anos, apresentou quadro de dor e distensão abdominal, náuseas \\ e vômitos, 15 dias após cirurgia videolaparoscópica, após exames de imagem, foi identificado encarceramento \\ da borda antimesentérica intestinal por meio da incisão de um dos portais laparoscópicos. Realizada \\ ressecção do segmento de delgado com endogrampeador e realizada anastomose entero-entero, latero- \\ lateral com endogrampeador, paciente evoluiu bem em pós-operatório. A hérnia de Richter é definida por um \\ tipo raro de hérnia na parede abdominal em que apenas uma parte da alça intestinal, a borda antimesentérica, \\ fica presa no orifício herniário, podendo ser estrangulada. Essas hérnias podem ocorrer em incisões \\ anteriores e se apresentam com clínica de dor abdominal, náusea, vômitos e raramente apresentam sintomas \\ obstrutivos, assim tendem a progredir mais rapidamente para gangrena. O tratamento geralmente é cirúrgico. \\ Considerações finais: Ainda que seja uma condição médica rara, deve-se ter um alto índice de suspeição, \\ principalmente em pós-operatório laparoscópico e em pacientes com quadro clínico abdominal vago.
}

Palavras-chave: Obstrução intestinal, Hérnia abdominal, Hérnia incisional.

\begin{abstract}
Objective: Presentation of the clinical picture of a patient who presented Richter's hernia, a rare condition. Case details: Patient, 64 years old, presented with abdominal pain and distension, nausea and vomiting, 15 days after videolaparoscopic surgery, after imaging exams, an incarceration of the intestinal antimesenteric border was identified through the incision of one of the laparoscopic portals. After resection of the small segment with an endostapler and enteroenteral, lateral-lateral anastomosis with an endostapler, the patient evolved well in the postoperative period. Richter's hernia is defined as a rare type of hernia in the abdominal wall in which only part of the intestinal loop, the antimesenteric edge, is trapped in the hernial orifice and can be strangled. These hernias can occur in anterior incisions and present with clinical symptoms of abdominal pain, nausea, vomiting and rarely present obstructive symptoms, thus tending to progress more rapidly to gangrene. Treatment is usually surgical. Final considerations: Although it is a rare medical condition, there must be a high index of suspicion, especially in the laparoscopic postoperative period and in patients with a vague abdominal clinical picture.
\end{abstract}

Key words: Intestinal obstruction, Abdominal hernias, Incisional hernia.

\section{RESUMEN}

Objetivo: Presentación del cuadro clínico de un paciente que presentaba una hernia de Richter, una entidad poco frecuente. Detalles del caso: Paciente de 64 años que presentó dolor y distensión abdominal, náuseas y vómitos, a los 15 días de la cirugía videolaparoscópica, luego de exámenes de imagen, se identificó una incarceración del borde antimesentérico intestinal a través de la incisión de uno de los portales laparoscópicos. Tras resección del segmento pequeño con endograpadora y anastomosis enteroenteral lateral-lateral con endograpadora, el paciente evolucionó bien en el postoperatorio. La hernia de Richter se define como un tipo raro de hernia en la pared abdominal en la que solo una parte del asa intestinal, el borde antimesentérico, queda atrapada en el orificio herniario y puede estrangularse. Estas hernias pueden ocurrir en incisiones

${ }^{1}$ Serviço de Cirurgia do Aparelho Digestivo do Hospital São Paulo de Muriaé, Muriaé - MG.

${ }^{2}$ Centro Universitário de Minas (UNIFAMINAS), Muriaé - MG. *E-mail: nataly_tolentino@hotmail.com

SUBMETIDO EM: 7/2021

ACEITO EM: 7/2021

PUBLICADO EM: 8/2021 
anteriores y presentarse con síntomas clínicos de dolor abdominal, náuseas, vómitos y rara vez presentan síntomas obstructivos, por lo que tienden a progresar más rápidamente a gangrena. El tratamiento suele ser quirúrgico. Consideraciones finales: Aunque es una condición médica rara, debe existir un alto índice de sospecha, especialmente en el postoperatorio laparoscópico y en pacientes con un cuadro clínico abdominal vago.

Palabras clave: Obstrucción intestinal, Hernia abdominal, Hernia incisional.

\section{INTRODUÇÃO}

Hérnia de Richter (HR) é uma patologia rara, sendo um subtipo de hérnia abdominal que resulta de um encarceramento de parte do intestino, a borda antimesentérica, em um orifício herniário, que pode estar localizado em qualquer topografia usual de hérnia, tendo como o local mais comum o canal femoral, seguida pelo canal inguinal e de hérnia incisional da parede abdominal e apresentando como conteúdo de hérnia mais comumente uma alça do intestino delgado (íleo distal), embora também possa apresentar como conteúdo, porém menos frequente, alças de intestino grosso, de omento, trompas de falópio ou até mesmo o apêndice (CHEN W, et al., 2017; BATISTA ABE, et al., 2019; HAYES C, et al., 2020.

Frequentemente, o intestino envolvido pelo anel herniário evolui para gangrena de forma rápida, pois 0 conteúdo herniado, a borda antimesentérica, possui uma circulação colateral pobre o que acaba prejudicando a sua vascularização e nutrição, podendo resultar em perfuração aumentando a gravidade do caso (BOHATCH MSJ, et al., 2018; BATISTA ABE, et al., 2019; HAYES C, et al., 2020; RAO SD e KANAGAVEL $\mathrm{M}, 2019)$.

Os sinais e sintomas costumam surgir de forma insidiosa e não típica, fazendo assim que exame clínico minucioso seja essencial para o diagnóstico precoce a fim de evitar complicações, embora seja necessária também à investigação com métodos de imagem complementares, como ultrassonografia, tomografia computadorizada, que é padrão ouro, e até angiografia por TC quando disponível (MARTIS JJ, et al., 2011; YOUSUF P, et al., 2016; JAYAMANNE H e STEPHENSON BM, 2017; KATRAGADDA K, et al., 2019; DINESH DS, et al., 2021).

Segundo Rao SD e Kanagavel M (2019) as HR são classificadas em três grupos, em que o primeiro foi chamado de 'grupo obstrutivo' caracterizado por náusea, vômito, peritonite e obstrução intestinal e alto risco de morte se não tratada, o segundo grupo foi chamado de 'grupo pós-necrótico', caracterizado por estrangulamento local com necrose e perfuração levando a fístula enterocutânea e o terceiro grupo foi chamado de 'grupo perigoso', que incluí sinais e sintomas leves, o que causou maior atraso no diagnóstico aumentando a morbidade e mortalidade.

Entre os diagnósticos diferenciais da hérnia femoral estão à pseudo-hérnia, o pseudoaneurisma da artéria femoral, a insuficiência venosa da veia safena, o tumor de partes mole e a linfadenopatia (BOHATCH MSJ, et al., 2018). O tratamento é cirúrgico via laparotomia exploradora, permitindo ressecção de alças se necessário, além de lavagem da cavidade em casos de peritonite e inspeção geral para exclusão de outros diagnósticos diferenciais (KATRAGADDA K, et al., 2019; YOUSUF P, et al., 2016; MARTIS JJ, et al., 2011).

Este trabalho teve como objetivo descrever essa condição rara, por vezes, de difícil diagnóstico, pois geralmente é oligossintomática, que pode acarretar em diagnóstico tardio com complicações graves e potencialmente letais.

\section{DETALHAMENTO DO CASO}

Paciente do sexo feminino, 64 anos, com antecedentes de histerectomia transvaginal há 30 dias, que evolui com dor abdominal no terceiro dia pós-operatório, apresentando hemorragia abdominal e repercussão hemodinâmica. Foi submetida à videolaparoscopia diagnostica que identificou sangue na cavidade, mas também ausência de sangramento ativo, optando-se por tratamento conservador. 
Ela recebeu alta hospitalar, porém permaneceu com dor, distensão abdominal, evoluindo com náuseas e vômitos importante. Duas semanas após a alta hospitalar, procurou o pronto atendimento, ao exame físico apresentava queda do estado geral, desidratada $+2 /+4$, hipocorada, anictérica, acianótica, eupneica em ar ambiente e afebril, exame físico cardiovascular sem alterações. Presença de edemas em membros inferiores $+2 /+4$. Abdome distendido difusamente e presença de abaulamento não redutível em região inguinal esquerda (região de portal da laparoscopia), doloroso a palpação e sem sinais flogísticos locais.

Foram solicitados exames laboratoriais, onde hemograma evidenciou leucócitos normais e sem presença de anemia, mas apresentou uma leve hiponatremia. Foi solicitada tomografia computadorizada de abdome com contraste que evidenciou hérnia incisional em flanco esquerdo, com insinuação de alça ileal, havendo importante dilatação de alças jejunais e ileais, com níveis líquidos e retardo no esvaziamento intestinal. Segmentos colônicos parcialmente distendidos, observando-se restos de contraste radiológicos (Figura 1).

Figura 1 - Tomografia Computadorizada.

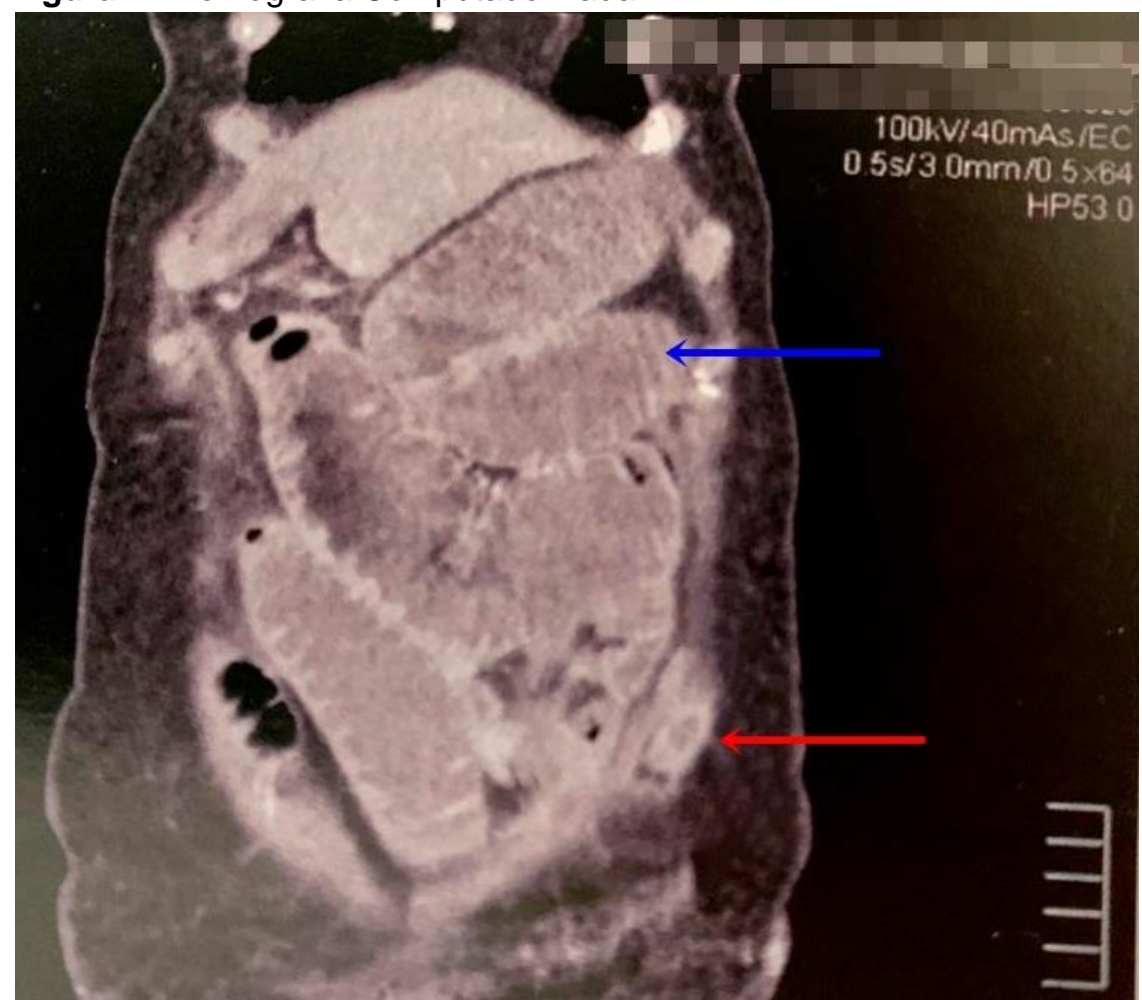

Legenda: Presença de insinuação de alça ileal através de hérnia incicional, Hérnia de Richter (seta vermelha) e distensão de alças (seta azul). Fonte: Pereira LD, et al., 2021.

A paciente foi submetida ao procedimento cirúrgico, realizada incisão transversa sobre cicatriz do portal de laparoscopia no flanco esquerdo. Achado operatório de encarceramento da borda antimesentérica do intestino delgado, característico de Hérnia de Richter. Foi realizada ressecção do segmento de delgado com endogrampeador, realizada anastomose entero-entero, latero-lateral com endogrampeador e limpeza de cavidade abdominal com soro fisiológico aquecido. Reforço com tela de malex $15,0 \times 15,0 \mathrm{~cm}$ sublay e fechamento por planos.

O pós-operatório foi conduzido em Unidade de Terapia Intensiva onde a paciente apresentou-se lúcida e orientada, colaborativa, eupneica com $\mathrm{O} 2$ no cateter nasal a $1 \mathrm{~L} / \mathrm{min}$, sem sinais de esforço respiratório, tosse seca, afebril, acianótica, evoluindo com distúrbios hidroeletrolíticos e anemia grave. A alimentação foi reintroduzida de forma gradativa, foi realizado correção hidroeletrolítica com cloreto de sódio e cloreto de potássio e transfusão sanguínea em Quinto dia do pós-operatório. Laudo anatomopatológico de alça de 
delgado evidenciou segmento de intestino delgado medindo $31,0 \mathrm{~cm}$ de comprimento e apresentando perímetro regular de $3,0 \mathrm{~cm}$, exibindo áreas de edema, hemorragia e presença de vasos ectasiados. Constatou-se ausência de sinais de malignidade. Com estabilidade hemodinâmica e boa evolução após o manejo clínico a paciente recebeu alta médica após 12 dias de tratamento hospitalar.

O presente relato foi submetido ao Comitê de Ética em Pesquisa (CEP), sendo aprovado com o parecer de número 4.822.527.

\section{DISCUSSÃO}

HR é definida como um tipo raro de hérnia que afeta a parede abdominal, na qual apenas uma parte da circunferência da alça intestinal, a borda mesentérica, fica presa na hérnia e estrangulada no orifício herniário (BATISTA ABE, et al., 2019; FOSTER D, et al., 2015).

O primeiro caso de HR foi observado por Fabricius Hildanus entre 1598 e 1606, de acordo com Batista ABE, et al. (2019), e a primeira descrição científica foi dada, por August Gottlieb Richter ou August Richter de Gottingenem entre 1778 e 1785, quando foi apresentada como "a pequena ruptura" e em 1887, Sir Frederick Treves foi quem propôs o título definitivo de "Hérnia de Richter" para a comunidade científica.

As HR são mais comumente diagnosticadas em pacientes do sexo feminino, representam cerca de $6 \%$ do total das hérnias estranguladas e podem ocorrer em qualquer idade, mas apesar disso, são mais comuns na idade entre 60 e 80 anos, conforme Katragadda K, et al. (2019), Rao SD e Kanagavel M (2019) e Georgios $\mathrm{S}$, et al. (2017), como pode-se ver no caso em questão, em que a paciente é do sexo feminino apresentava 64 anos no dia do diagnóstico do quadro. Essas hérnias podem ocorrer tanto por defeito na parede abdominal como as demais hérnias, quanto em incisões de cirurgias anteriores, e são mais comumente vistos em pequenos anéis de hérnia com tamanho suficiente para prender uma pequena porção de a parede do intestino (KATRAGADDA K, et al., 2019).

De acordo com Batista ABE, et al. (2019), a apresentação clínica tem quadros abdominais confusos de dor, náusea e vômitos simulando quadro clínico de gastroenterite, o achado físico mais constante é sensibilidade ou edema sobre o orifício da hérnia. A paciente do caso relatado apresentou quadro clínico semelhante, e por ter sido submetida à cirurgia videolaparoscópica recentemente, a hipótese de Hérnia de Richter foi levantada. Como os pacientes apresentam sintomas vagos e raramente obstrutivos, a HR se torna difícil de serem diagnosticados antes de ocorrer o estrangulamento da alça envolvida, e assim esses pacientes tendem a progredir mais rapidamente para gangrena do que normalmente é visto em outros tipos de hérnias estranguladas.

O intestino necrótico não é o único aspecto preocupante dessas hérnias, incluindo outras complicações como perfurações da alça estrangulada, que gera peritonite aguda, fístulas espontâneas do intestino afetado ou fístulas enterocutâneas, abscesso de parede abdominal e de cavidade (GURMEET SS, 2019; BATISTA ABE, et al., 2019). Segundo Gurmeet SS (2019) e Georgios S, et al. (2017) o ultrassom e a tomografia computadorizada assumem um trabalho notável no diagnóstico, auxiliando no manejo pré-operatório e diagnóstico diferencial de massa abdominal ou abscesso.

As hérnias incisionais da parede abdominal compreendem cerca de $4-25 \%$ das $\mathrm{HR}$ de acordo com Regelsberger-Alvarez CM e Pfeifer C (2020), podendo a incidência de hérnias de trocanter variar de $0,2 \%$ a 3,1\% (EMRE ETAŞ, et al., 2017). O aumento dos procedimentos laparoscópicos e assistidos por robótica levou ao aumento desse tipo de hérnia (GEORGIOS S, et al., 2017; REGELSBERGER-ALVAREZ CM e PFEIFER C, 2020). Sendo que o tamanho e os locais dos trocartes laparoscópicos, especialmente de 10-20 $\mathrm{mm}$, formam o ambiente ideal para o desenvolvimento de uma hérnia de Richter (KHUROOUMA S, et al., 2021).

O fechamento incompleto da fáscia no local do trocanter, trocanter da linha média, alongamento do local da porta para recuperação de órgãos, efeito de vácuo durante a retirada da porta, defeitos fáscias preexistentes, obesidade, idade avançada, má nutrição e infecção no local da operação são alguns dos 
fatores comuns associados ao desenvolvimento dessas hérnias (EMRE ETAŞ, et al., 2017; SAFI AF, et al., 2016; BATISTA ABE, et al., 2019).

De acordo com Memon ZA, et al. (2019) e lbáñez M, et al. (2020), as características mais determinantes para o desenvolvimento desta hérnia são o tamanho do orifício herniário e sua consistência, está por sua vez, evita que a pressão exercida pelo conteúdo herniário permita a passagem de toda a alça para seu interior, já o orifício herniário deve ter um diâmetro grande o suficiente para ser atravessado pela parede da alça intestinal, mas não permitir sua passagem completa, e ele possui grande importância também pelo no fato de se relacionar com maior risco de isquemia intestinal, aumentando esse risco em comparação para os outros encarceramentos herniários, justificado pela pobre vascularização da borda, e com consistência que.

As HR possuem uma taxa de mortalidade significativamente alta de $17 \%$ a $21 \%$, podendo chegar a $60 \%$ em casos de necrose e perfuração da alça, sendo o prognóstico particularmente desfavorável se peritonite (YOUSUF P, et al., 2016; BOHATCH MSJ, et al., 2018). É uma condição médica que requer tratamento cirúrgico urgente, tanto para evitar a necrose e a perfuração, o que causa piora do prognóstico, quanto para exclusão dos diagnósticos diferenciais, e devido às suas possíveis complicações e esse tratamento depende do grau de lesão isquêmica na parede intestinal, logo deve ser realizando um exame cuidadoso e paciente da alça intestinal encarcerada para verificar sua viabilidade, e se o intestino for viável pode ser reduzido e o reparo do defeito herniário realizado por herniorrafia ou hernioplastia com tela sendo o ideal (GEORGIOS S, et al., 2017; JAYAMANNE H e STEPHENSON BM, 2017; FOSTER D, et al,. 2015; DINESH DS, et al., 2021).

Entretanto, a necrose e as possíveis complicações são o motivo de a ressecção intestinal ser obrigatória, chegando a ser realizada em cerca 50\% dos casos (KATRAGADDA K, et al., 2019; YOUSUF P, et al., 2016; GURMEET SS, 2019; RUIZ-FUNES MAP, et al., 2020). De acordo com Batista ABE, et al. (2019) e RuizFunes MAP, et al., (2020) quando há necrose e infecção no defeito anatômico da parede, ou seja, no orifício herniário é melhor drenar toda a cavidade ou ou deixa-la aberta e depois, e um segundo momento, voltar para reparar a hérnia. Em outros casos, onde não há perfuração e pequena área de necrose da alça intestinal que não se estende até a borda do mesentério, pode ser realizadas suturas invaginadas no local.

Segundo Ruiz-Funes MAP, et al. (2020), a vantagem de inventário da cavidade abdominal, descarta-se pela possibilidade de exclusão de outras patologias ou evidências de complicações, com possibilidade terapêutica sob a mesma abordagem.

A paciente encontra-se no padrão mais comum de diagnóstico, devido à identificação precoce e à intervenção cirúrgica oportuna, evitou-se a perfuração intestinal, o que contribuiu para uma melhor evolução do seu quadro. Ainda que seja uma condição médica rara, deve-se ter atenção e um alto índice de suspeição, e levando em consideração o fato do aumento de procedimentos videolaparoscópicos, a história pregressa do paciente torna-se importante, principalmente pelo fato de poder mimetizar complicações mais benignas, como hematomas no local do portal de laparoscopia. A tomografia é um excelente exame diagnóstico. $O$ manejo cirúrgico oportuno é essencial tanto para evitar a necrose e a perfuração. Neste caso, o cirurgião optou por realizar a ressecção de delgado, evitando maiores danos para a paciente.

\section{REFERÊNCIAS}

1. BATISTA ABE, et al. Richter's hernia: case report. Revista de Saúde. 2019; 10(2): 66-70.

2. BOHATCH MSJ, et al. Hérnia femoral de Richter. Relatos Casos Cir. 2018; (4): e1902.

3. CHEN W, et al. Relato de caso de fístula enterocutânea umbilical espontânea resultante de hérnia de Richter encarcerada, com breve revisão da literatura. BMC Surg. 2017; 17(1): 15/s12893-017-0216-z.

4. DINESH DS, et al. Richter-type of spigelian hernia: A rare case report. Int J Surg Sci 2021; 5(1): 288-289.

5. EMRE ETAŞ, et al. Richter's hernia after gynecological laparoscopic procedure: a case report. Medical Journal of Islamic World Academy of Sciences 2017; 25(1): 22-24.

6. FOSTER D, et al. Richter-type Spigelian hernia: A case report and review of the literature. International Journal of Surgery Case Reports, Volume 6, 2015, Pages 160-162.

7. GEORGIOS S, et al. Strangulated richter's incisional hernia presenting as an abdominal mass with necrosis of the overlapping skin. A case report and review of the literature. Clinical Case Reports 2017; 5(3): 253-256.

8. GURMEET SS. Richter's Hernia: A Case Report. Prac Clin Invest, 2019; 2(1): 27-30 
9. HAYES C, et al. Obturator hernia of Richter type: a diagnostic dilemma. BMJ Case Reports CP, 2020; 13 : e238252.

10. IBÁÑEZ M, et al. Fístula intestinal posoperatoria tras herniorrafía umbilical por hernia de Richter insospechada. Rev Hispanoam Hernia. 2020; 8(2): 89-92.

11. JAYAMANNE H, STEPHENSON BM. Herniação de Richter perineal após proctectomia. Ann R Coll Surg Engl. 2017; 99 (8): e244-e245.

12. MARTIS JJ, et al. Strangulated Richter's Umbilical Hernia - A Case Report. Indian J Surg. 2011; 73(6): $455-7$.

13. KATRAGADDA K, et al. A case of richter hernia: a rare entity. American College of Surgeons - ACS Case Reviews. 2019; 2(3): 5-7.

14. KHUROOUMA S, et al. Hérnia de Richter incomum: corpo estranho impactado que leva ao encarceramento e perfuração - uma entidade clínica rara. Internacional Diário de Cirurgia Caso Relatórios. 2021; 79.

15. MEMON ZA, et al. Caso raro de peritonite devido a perfuração ileal secundária ao tipo de hérnia obturadora de Richter. Cureus. 2019; 11 (3): e4289.

16. RAO SD, KANAGAVEL M. Recurrent Inguinal Hernia as Richter's Hernia. Surg Med Open Acc J. 2019; 3(1).

17. REGELSBERGER-ALVAREZ CM, PFEIFER C. Richter hernia. StatPearls, Treasure Island (FL), 2020.

18. RUIZ-FUNES MAP, et al. Abordaje de hernias poco frecuentes por cirugía de mínimo acceso: serie de casos. Rev Mex Cir Endoscop. 2020; 21(1): 6-14.

19. SAFI AF, et al. Influência do volume de hérnia de tecido mole nos sintomas clínicos de pacientes com fraturas do assoalho da órbita. J Craniomaxillofac Surg. 2016; 44(12): 1929-1934.

20. YOUSUF P, et al. Richters hernia-a rare phenomenon in surgery. IOSR Journal of Dental and Medical. 2016; 15(9): 35-36. 